\title{
Notes de lecture sur \\ Les sciences du langage et la question de l'interprétation (aujourd'hui), Éditions Lambert-Lucas, 2018, 258 pp.
}

Janetta-Daniela BĂRĂITARU1

\begin{abstract}
This article is a proposal for reading the book "Les sciences du langage et la question de l'interprétation (aujourd'hui)»
\end{abstract}

Keywords: interpretation; comprehension; linguistics; analysis DOI: $10.24818 /$ DLG/2021/38/17

$\mathrm{M}$ ettant sur le devant de la scène les notions, fondamentales en linguistique, d'interprétation et de compréhension, le livre "Les sciences du langage et la question de l'interprétation (aujourd'hui) » est une collection de contributions au Colloque de l'Association des Sciences du Langage de 2017, contributions qui relèvent de domaines variés tels la sémiotique, la sémantique référentielle et lexicale et la pragmatique argumentative ou interactionnelle, la linguistique analogique ou l'analyse textuelle du discours. En partant de l'idée que l'interprétation dans les sciences du langage ne réunit pas le consensus des linguistes, les auteurs du chapitre de présentation du livre, Guy Achard-Bayle, Maximilien Guérin, Georges Kleiber et Marina Klylyschin en analysent les raisons externes et internes et tracent un parcours historique qui remonte jusqu'à Aristote et Platon. Les spécialistes se posent ensuite des questions sur la place faite au processus interprétatif dans différents cadres épistémologiques, sur les relations entre les notions spécifiques liées à l'interprétation, le traitement des ambiguïtés, la nature du processus interprétatif, la dynamique interprétative en fonction de l'ordre des signifiés ou de la relation entre signifiants. C'est à ces interrogations et à d'autres encore que les articles publiés dans le livre essaient de répondre.

\footnotetext{
${ }^{1}$ Janetta-Daniela Baraitaru, doctorande, Université “Ovidius" de Constantza,
} Roumanie, jbaraitaru@yahoo.com 
Sans être organisées par chapitres ou par thèmes, les contributions ont comme point commun le fait qu'elles émanent de spécialistes du domaine, qui posent le problème des entraves des approches existantes sur l'intreprétation. Certains titres contiennent des termes qui renvoient dès le début aux difficultés posées par le processus interprétatif : "écueils », " limites », « ambiguïté », " marges », " variation », « mésinterprétation ». L'ordre dans lequel apparaissent les articles permet au lecteur de parcourir un itinéraire très complexe et éclaircissant sur cette problématique, ayant comme point de départ l'analyse des définitions données par les dictionnaires au terme d'interprétation et le parallèle avec d'autres notions comme la compréhension, le sens, la signfication, pour arriver à des analyses très appliquées sur des textes poétiques ou argumentatifs, des discours politiques, des conversations.

Dans « Compréhension et interprétation: Interrogations autour de deux modes d'appréhension du sens dans les sciences du langage » (pp. 21-54), Patrick Caraudeau s'interroge sur les notions de compréhension et d'interprétation, en partant de l'idée qu'on ne peut pas les traiter sans se rapporter à la tradition de l'herméneutique, mais en $\mathrm{s}^{\prime}$ autorisant à repenser la perspective sur celles-ci dans le cadre des sciences du langage. Après avoir analysé les définitions des dictionnaires pour « comprendre »/ «compréhension » et " interpréter » / " interprétation », l'auteur arrive à la conclusion que les premières opérations correspondent à " un acte global résultatif », tandis que les secondes - à « une série d'opérations ». Il essaie ensuite d'établir la distinction entre les deux notions dans la perspective de l'herméneutique, démarche très difficile à mener à bon fin; c'est l'occasion de se demander ce que les sciences du langage peuvent emprunter à ce domaine et de lancer la discussion sur l'opposition entre le sens et la signification dans l'herméneutique et la linguistique. En mettant en relation ces concepts avec la compréhension, Patrick Charaudeau constate qu'il existe une compréhension spécifique $d u$ sens (littérale et objective, de l'ordre du plausible) et une compréhension de la signification (indirecte, tenant au contexte de production / réception de l'acte de langage). L'auteur traite aussi des opérations interprétatives par divers types d'inférences et d'interprétations selon le statut du sujet interprétant. Il se déclare d'accord dans sa conclusion avec Nietzche, qui percevait l'interprétation non comme un résultat, mais comme un processus, et lance un nouveau débat, sur ce que devrait être la posture du chercheur ou du critique dans l'interprétation. 
Catherine Kerbrat-Orecchioni, dans « La Méduse apprivoisée: l'analyse du discours » (pp.55-78), part des préliminaires sur la relation entre le sens et l'interprétation et sur la question des observables en analyse du discours. L'auteure présente ensuite les principes de l'interprétation en analyse du discours en interaction: décrire un échantillon de discours signifie interpréter, action qui signifie à son tour essayer la reconstitution des interprétations des participants, identifier la place de l'emetteur dans l'interprétation, voir l'analyste comme un « archi-interprétant». Ce dernier doit annoncer au préalable, selon l'auteure, la nature exacte de l'objet et l'objectif de l'analyse, qui tiennent au texte, mais aussi au contexte. Ainsi y a-t-il différence $d$ 'interprétation entre un discours monologal (tels une conférence ou un article de presse) et un discours dialogué (tel le débat présidentiel). Le bilan synthétise les conditions nécessaires pour une interprétation " recevable » et définit la conduite de l'analyste, " qui consiste à admettre une certaine pluralité d'interprétations mais fortement contrainte par ces garde-fous qui sont les diverses composantes de la matière signifiante, que l'analyste se doit d'observer, sans s'illusionner sur leur transparence ni se laisser méduser par leur opacité » (p. 76).

Dans « De la construction du sens à la pragmatique interprétative » (pp. 79-95), Jacques Fontanille part de l'hypothèse que si on réussit à spécifier la pratique de l'interprétation, on parvient à en rendre compte dans une analyse sémiotique. L'auteur présente le concept d'interprétation en relation avec les différentes perspectives sémiotiques proposées au fil du temps par Greimas, Peirce, Saussure, Eco ou Rastier. En partant des deux acceptions du terme "interprétation», celle de sélectionner l'une des significations possibles d'un texte et jouer d'un instrument, l'auteur considère que celle-ci se manifeste obligatoirement «par la production d'une sémiotique-objet, donc une sémiose (donc une énonciation et un énoncé), qui doit être dans un mode sémiotique différent de ce sur quoi elle porte. » (p.84). En plus, l'auteur considère que l'analyse de l'interprétation ne doit pas etre confondue avec la praxis énonciative ( l'ensemble des actes par lesquels des discours sont convoqués, sélectionnés, manipulés et inventés par chaque énonciation particulière », p. 87). Dans le contexte de cette distinction, la pratique interprétative participe à la praxis énonciative et peut être l'objet d'une approche sémiotique, donc elle peut elle-même être soumise à la construction du sens. La conclusion est que «la signification de l'acte d'interprétation se déploie [...] sur des dimensions pratiques, rhétoriques, éthiques et anthroposémiotiques » (p. 94). 
Marianne Doury présente dans son article, «Les écueils de l'interprétation de l'argumentation »(pp.97-126), après un passage en revue des principes de l'interprétation des données argumentatives, les résultats de l'analyse de 52 copies de ses étudiants, remises lors des examens de validation d'un cours d'argumentation, en 2016 et 2017. L'analyse porte sur les réponses à la première question de l'épreuve, supposant plusieurs tâches à accomplir sur un texte donné : spécifier la position défendue dans le texte, identifier les lieux d'introduction et les modes de traitement $\mathrm{du}$ contre-discours, identifier plusieurs types d'arguments etc. Outre les problèmes de compréhension de la consigne, l'auteure découvre plusieurs difficultés dans l'interprétation de l'argumentation, telles l'erreur sur la position défendue, due aux problèmes de compétence lexicale, des erreurs de détection des axiologies ou l'(in)sensibilité aux mécanismes énonciatifs et polyphoniques. Il existe aussi des erreurs dans le repérage des séquences argumentatives et dans l'dentification du type d'argument, à cause de la mauvaise interprétation des indicateurs ou de l'influence du nom du type d'argument. Ensuite, elle construit un exemple d'analyse sur l'un des textes proposés aux étudiants, le discours de Robespierre prononcé le 30 mai 1791 à la Constituante, en guise de modèle. La conclusion de Marianne Doury ouvre une autre piste de discussion, celle sur « le gouffre » qui existe entre l'interprétation de l'analyste (étudiants, elle-même) et celle des locuteurs participants aux échanges «qui font sens autant par leur capacite à rendre justice aux argumentations auxquelles ils sont confrontés que par les effets qu'ils produisent dans l'interaction » (p.120).

Construit sur l'interprétation d'un autre type de texte, l'article « Interpréter le poème: une interaction variable entre trois dimensions textuelles (sémantique, esthétique et énonciative)» de Michèle Monte s'occupe des difficultés rencontrées dans l'interprétation de la poésie du point de vue linguistique. L'auteure commence en lançant une question fondamentale: "Quels outils conceptuels pour définir la poéticité ?», question dont la réponse contient des éléments repris de l'Antiquité grécolatine, Jakobson, Dominicy ou Rastier (p.128). Ensuite, l'auteure présente les trois dimensions $\mathrm{du}$ texte et leur interaction dans le poème (la dimension sémantique, esthétique et énonciative), qui n'est pas propre seulement aux textes littéraires, mais à tout type de production textuelle, et propose un parcours interprétatif de trois poèmes appartenant à la poésie lyrique (un sonnet de Ronsard, un poème des Contemplations d'Hugo et 
un poème de Réda). Elle réussit à en dégager les trois dimensions de son modèle, «à la fois interreliées et partiellement indépendantes » (p.147). À la fin de son parcours, Michele Monte précise que la prise en compte dans une analyse ultérieure du contexte socio-historique ou de l'appartenance générique des poèmes analysés pourrait enrichir et même modifier l'interprétation.

De son côté, Richard Trim propose une approche contrastive dans « L'influence de la morphologie sur l'interprétation des images doubles au sein des métaphores littéraires »(pp.155-170). Il s'agit, plus particulièrement, de souligner le rôle de la morphosyntaxe par rapport aux mots composés. L'auteur part du constat que la façon variable de construction des mots composés dans certaines des langues européennes (germaniques et romanes) pourrait influencer leur conceptualisation, mais aussi leur interprétation. L'auteur essaie de démontrer que les mots composés (noms, adjectifs) peuvent avoir plus de force que les comparaisons dans les textes littéraires, en s'appuyant sur des études faites dans le domaine ainsi que sur des exemples contrastifs provenant de l'anglais, de l'allemand et du français. Sa conclusion est que "Même si le sens d'une métaphore composée peut être transmis par une autre structure figurée telle une comparaison [...] la force stylistique d'un composé reste plus élevé dans les discours littéraires [...]. » (p. 168) et que les différences morphosyntaxiques entre les langues peuvent influencer le degré et l'étendue de l'interprétation.

Dans «Les limites de l'interprétation à lumière de l'analogie » (pp. 171-195), Philippe Monneret, comme Patrick Charaudeau dans son texte, part de l'idee que la compréhension et l'interprétation sont deux parties distinctes d'un seul processus, à l'intérieur duquel la première découle de la seconde, et qu'il existe des cas paradoxaux de compréhension sans interprétation. Pour répondre à la question lancée dès le début : " Comment et pourquoi peut-on comprendre sans interprétation ? »(p.173), l'auteur présente d'abord le cadre général de son analyse - la linguistique théorique (structurée par trois approches différentes : cognitive, sémiotique et logique) et le processus d'analogie (vu dans la perspective psychologique et linguistique). Il s'occupe ensuite de la compréhension avec ou sans interprétation à la lumière des fonctions de l'analogie (catégorisatrice, iconique, figurative, argumentative, paraphrastique), à travers l'analyse d'exemples comme des énoncés, des images, des métaphores, des traductions. Ainsi découvre-t-il trois cas de 
compréhension sans interprétation: celui où la sensation fait jouer seulement la perception, l'attitude descriptive et les énoncés parfaitement contextualisés. Il en conclut que "les situations de compréhension sans interprétation sont celles dans lesquelles aucune difficulté de compréhension ne se présente à l'allocutaire (pas d'ambiguïté sémantique, pas d'indétermination contextuelle). Dans ce cas, en comprenant, l'allocutaire réagit immédiatement à l'énoncé produit, sans distanciation à l'égard de cet énoncé et sans être nécessairement en mesure de justifier sa réaction. »(p.192). L'auteur ouvre son argumentation sur la nécessité d'étudier en détail les conditions de possibilité de l'interprétation, anlayse qu'il considère très différente de celle du processus interprétatif.

Dans l'article « Ambigüité et marges d'interprétation en synchronie et en diachronie lexicales : entre innovation et mésinterprétation » (pp. 197232), Esme Winter-Froemel part de la constatation qu'il y a des cas de divergences entre l'interprétation de l'émetteur et celle du récepteur, mais sans que le locuteur s'en rende compte nécessairement. L'auteure présente tout d'abord les repères sémiotiques de la communication, dans le but d'offrir un modèle, un cadre unifié et interdisciplinaire en vue d'étudier les différents types de divergences interprétatives. En analysant les reherches faites sur l'ambigüité, l'auteure souligne qu'il existe des cas très différents, au niveau du système de la langue et au niveau du discours ou de la parole, et met en évidence l'existence d'une série de douze paramètres pour décrire les cas d'ambigüité, qui se regroupent selon les situations de description sémiotique et sémantique, interactionnelle et énonciative ou de la dimension diachronique de celle-ci. À travers les analyses qui exploitent la combinaison entre les douze paramètres de l'approche sémiotique, l'auteure illustre six cas de figures emblématiques de divergences interprétatives manifestées dans la communication : la non-compréhension, les malentendus classiques ou les accidents communicationnels, les malentendus stratégiques, l'ironie, la politesse verbale et la réanalyse. Après un examen de l'évolution diachronique des divergences interprétatives, l'auteure propose de nouvelles pistes de recherche, sur les innovations potentielles observées en ce qui concerne la politesse verbale, l'ironie et l'analyse, et sur les types de contexte, considérés très importants dans l'analyse de l'évolution diachronique.

Enfin, dans le dernier article, "Stabilité sémantique et variation interprétative » (pp. 233-255), Georges Kleiber se donne comme objectif de «mettre en relief les variations interprétatives qui ne constituent pas un 
changement de sens lexical »(p.233), dans son intention d'aborder la variation interprétative non polysémique. La première partie de l'article est dédiée aux observations sur la polysémie et le sens multiple, qui suscitent des interrogations essentielles sur ce qu'on appelle pluralité des sens et sur ce qu'on entend par les sens pas totalement disjoints et une analyse de la variation interprétative. L'auteur dégage dix conclusions, qui permettent une meilleure compréhension des notions de variation interprétative et de sens multiple qu'on peut appliquer à une unité lexicale. Il passe ensuite, dans la deuxième partie, à la démonstration du fait qu'il n'y a pas toujours changement de sens quand il y a changement de référent (si l'unité lexicale connaît une variation référentielle due à son sens, si on fait porter à une unité lexicale le poids sémantique ou référentiel de l'expression dont elle est un constituant et dans les cas de polysémie systématique ou régulière, ou de métaphore conceptuelle ou généralisée). La troisième partie contient la démonstration qu'il n'y a pas toujours changement de référent en présence de la variation interprétative, fait qui s'explique à travers le principe sémiotique général de la métonymie intégrée.

Même si l'objectif du colloque n'était pas d'aplanir ou d'unifier la notion d'interprétation, nous pensons que le recueil présente une cohérence dans sa complexité et peut susciter autant l'intérêt des spécialistes que des débutants dans le domaine. Sans avoir donné une définition du concept qui soit acceptée par tout le monde, les contributions à l'ouvrage « Les sciences du langage et la question de l'interprétation (aujourd'hui) » se compose à comprendre le processus interprétatif dans le cadre théorique et dans le champ de l'analyse interprétative de divers types de discours. Elles apportent des réponses pertinentes, actuelles aux questions lancées au début du volume par les auteurs du livre et proposent également de nouvelles pistes pour l'étude approfondie des problématiques identifiées.

\section{Bibliographie}

1. Guy ACHARD-BAYLE et al., dirs, " Les Sciences du langage et la question de l'interprétation (aujourd'hui). Actes du colloque 2017 de l'Association des Sciences du Langage », Limoges, Éditions Lambert-Lucas, 2018. 